\title{
Follicular Carcinoma Arising from the Pyramidal Lobe of the Thyroid
}

\author{
Chie Ogawa ${ }^{1}$, Makoto Kammori ${ }^{1}$, Hiroyuki Onose ${ }^{1}$, \\ Emiko Yamada ${ }^{1}$ Kazuo Shimizu ${ }^{2}$ and Tetsu Yamada ${ }^{1}$ \\ ${ }^{1}$ Kanaji Thyroid Hospital, Tokyo \\ ${ }^{2}$ Department of Biological Regulation and Regenerative Surgery, Graduate School of Medicine, Nippon Medical School
}

\begin{abstract}
We present a rare case of follicular carcinoma arising from the pyramidal lobe of the thyroid in a 21-year-old woman. Radical resection of the thyroid isthmus was performed, followed by adjuvant hormonal therapy with levothyroxine. After 15 months of follow-up, the patient remains disease-free. Thyroid carcinoma in children and adolescents is rare, and also rarely arises in the pyramidal lobe. To our knowledge, this is the first report of this type of neoplasm arising from the thyroid pyramidal lobe. We are following up this case carefully, and if recurrence or metastasis or both occur, we plan to perform total thyroidectomy and ablation with ${ }^{131} \mathrm{I}$. This case suggests the importance of the differential diagnosis of midline cervical masses and the management of this type of neoplasm in adolescents.
\end{abstract}

(J Nippon Med Sch 2009; 76: 169-172)

Key words: follicular carcinoma, thyroid, pyramidal lobe

\section{Introduction}

The thyroid pyramidal lobe represents the inferior part of the thyroglossal duct and arises from the isthmus of the thyroid gland. Although the pyramidal lobe is considered a common thyroid lobe, it has been reported to be present in only about half of all individuals ${ }^{1,2}$. If the pyramidal lobe is present, it is generally excised during standard thyroid lobectomy for thyroid carcinoma. Sturniolo et al. have described the frequency and morphology of pyramidal lobe cancer in an Italian population, but their series included many cases of cancer that had invaded the pyramidal lobe from the main thyroid lobe ${ }^{3}$. Here we report what we believe to be the first recorded case of a neoplasm arising from the thyroid pyramidal lobe. The patient had first become aware of a cervical mass at the age of 12 years. Thyroid carcinoma in children and adolescents is also rare, papillary carcinoma being the most frequent histopathological subtype, and follicular carcinoma accounts for fewer than $20 \%$ of these cases. Here we report a rare case of follicular carcinoma of the pyramidal lobe of the thyroid in an adolescent patient.

\section{Case Report}

A 21-year-old woman was referred to our hospital in October 2007 with a chief complaint of an anterior cervical mass. She had first noticed the mass 9 years earlier. Although the patient had been followed up at another hospital, she was referred to us because of rapid enlargement of the mass in the previous 2 months. Physical examination revealed only a hard nodule in the anterior part of the neck (Fig. 1). Ultrasonography revealed an isoechoic solid mass

Correspondence to Makoto Kammori, MD, PhD, Kanaji Thyroid Hospital, 1-5-6 Nakazato, Kita-ku, Tokyo 114-0015,

Japan

E-mail: kanmori-dis@umin.ac.jp

Journal Website (http://www.nms.ac.jp/jnms/) 


\section{Ogawa, et al}

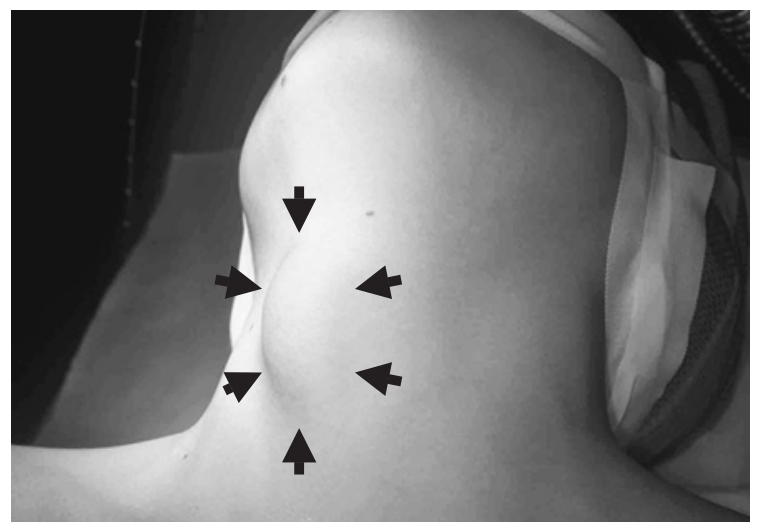

Fig. 1 A 21-year-old woman with follicular carcinoma of the thyroid. She was referred to our hospital with a chief complaint of an anterior cervical mass $(40 \mathrm{~mm}$ in size, indicated by black arrows).

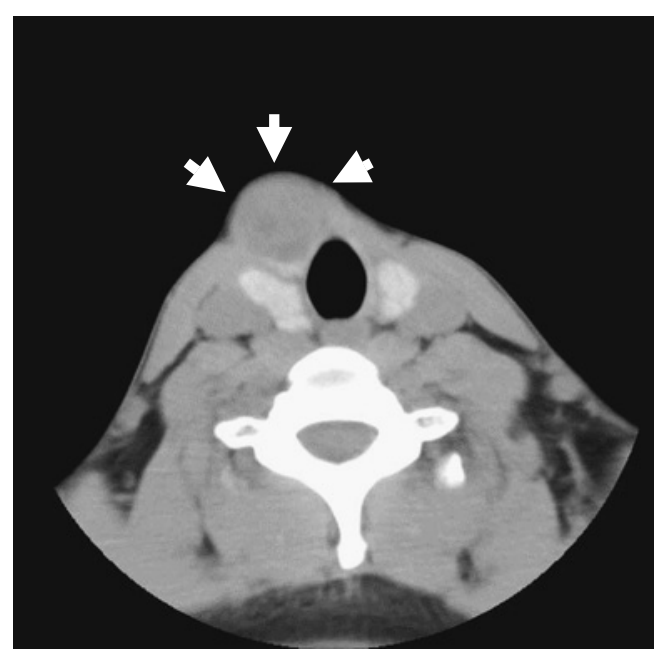

Fig. 2 Cervical computed tomography shows a low-density nodule (indicated by white arrows) with a capsule in contact with the pyramidal lobe of the thyroid.

with a capsule measuring $40.3 \times 27.0 \times 17.6 \mathrm{~mm}$ in the pyramidal lobe of the thyroid gland adjacent to the right lobe. Computed tomography revealed a round, low-density mass in contact with the pyramidal lobe anterior to the upper right thyroid lobe without swelling of the cervical lymph nodes. The margin of the mass was regular without evident invasion into the surrounding tissue (Fig. 2). Thyroid scintigraphy with ${ }^{201} \mathrm{Tl}$ demonstrated accumulation in the nodule and dilution with washout. Fine-needle aspiration biopsy indicated class IIIb (suspected follicular cancer). The serum thyroglobulin level was elevated to $1,404 \mathrm{ng} / \mathrm{mL}$ (normal range, $<32.7 \mathrm{ng} / \mathrm{mL}$ ). Levels of thyroid functional hormones were normal.

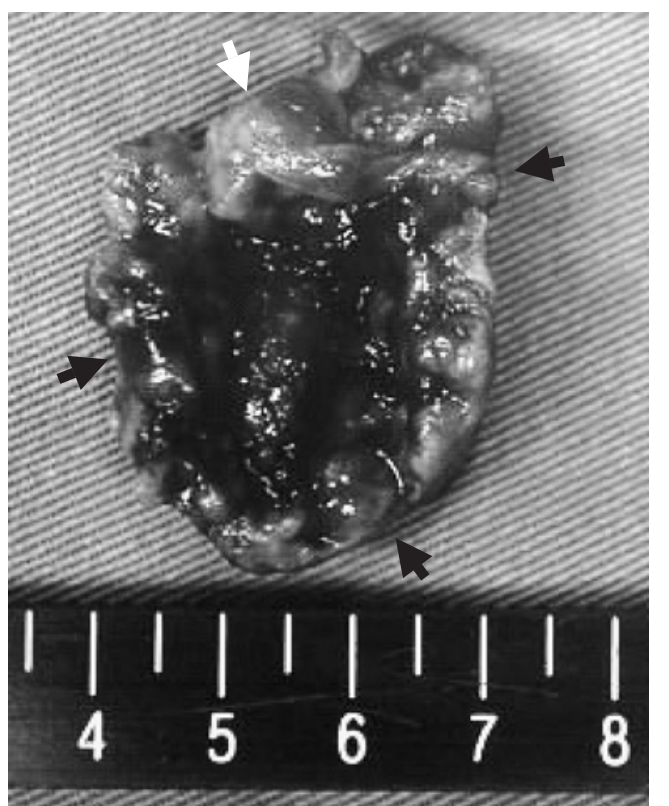

Fig. 3 Follicular carcinoma (indicated by black arrows) arises from the top of the pyramidal lobe of the thyroid (indicated by white arrow).

Because surgery was considered necessary owing to the size and continued enlargement of the tumor, the patient was admitted to our hospital and underwent surgery in November 2007. At surgery, the tumor was found to have arisen from the top of the pyramidal lobe of the thyroid (Fig. 3). We excised the tumor with the pyramidal lobe and the whole of the thyroid isthmus, with prelaryngeal lymphadenectomy. Histopathological examination revealed crowded development of small to middlesized follicular cells showing local invasion beyond the capsule and into blood vessels, without evidence of the thyroglossal duct (Fig. 4). The postoperative pathological diagnosis was a minimally invasive follicular carcinoma about $35 \mathrm{~mm}$ in diameter. There was no evidence of lymph node metastasis. The patient was discharged from our hospital with no complications and after 15 months of follow-up she remains disease-free. The serum thyroglobulin level has returned to normal $(10.0 \mathrm{ng} / \mathrm{mL})$, although the level of thyroid hormone did not change after the operation. We are observing this case carefully on an outpatient basis.

\section{Discussion}

Embryologically, the thyroid gland arises from a midline endodermal invagination of the foregut at the future foramen cecum and descends to its 

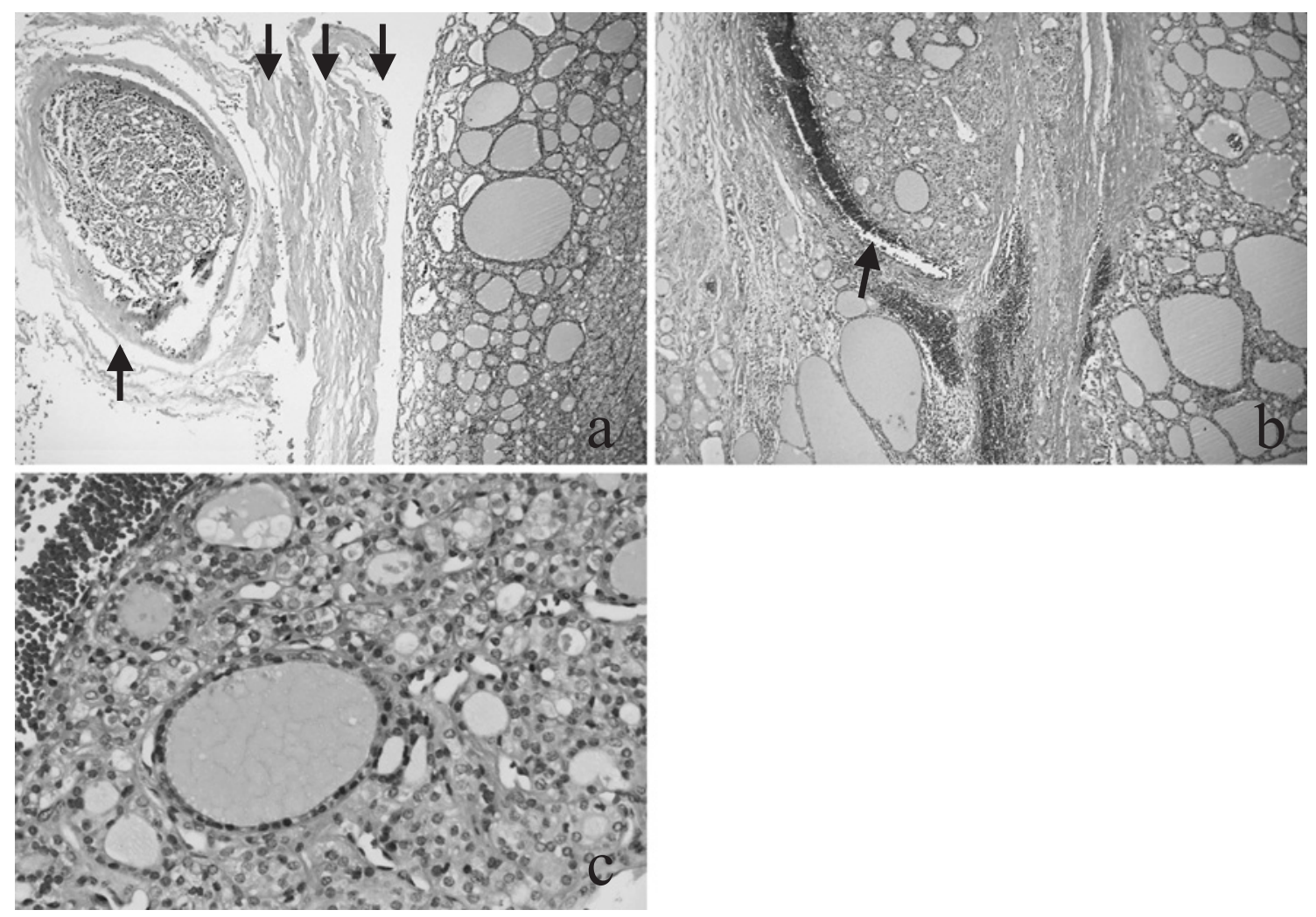

Fig. 4 a. Follicular carcinoma is observed to have a capsule (indicated by down arrows) and to have invaded blood vessels (indicated by up arrow, $\times 40$, low magnification). b. Follicular carcinoma is seen to have invaded beyond the tumor capsule (indicated by black arrow, $\times 40$, low magnification). c. Small to middle-sized follicular cells grow in crowds $(\times 200$, high magnification).

normal adult location through the thyroglossal duct by the 7th to 8th week of development. During the 9th to 10th week of development, the thyroglossal duct disappears. The pyramidal lobe represents the inferior part of the thyroglossal duct and is regarded as a normal component of the thyroid gland. However, the pyramidal lobe is not present in all individuals and has some anatomical variants. Braun et al. examined 60 cadavers and reported that the incidence of the pyramidal lobe was $55 \%^{1}$. In addition, branching from the left part, the right part, and the midline of the isthmus was present in $50 \%$, $12 \%$, and $28 \%$ of cases, respectively. The pyramidal lobe was present more frequently in men than in women. In the present patient, the pyramidal lobe, from which the carcinoma had arisen, had developed from the right part of the isthmus. Tumors arising in the pyramidal lobe are rare. In our hospital, this was the first case of carcinoma arising from the pyramidal lobe in 778 surgically treated patients with thyroid carcinoma (papillary carcinoma, 680 cases; follicular carcinoma, 63 cases; medullary carcinoma, 9 cases; undifferentiated carcinoma, 12 cases; and malignant lymphoma, 14 cases) from 1993 through 2008. An Italian review by Sturniolo et al. has described 44 cases of thyroid carcinoma associated with the pyramidal lobe among 1,405 patients who underwent surgery ${ }^{2}$. However, they showed that the primary disease invariably arose from the main gland. In the present case, follicular carcinoma originated from the pyramidal lobe, and no disease was evident in either lobes or the isthmus of the thyroid. Further histological examination indicated no sign of the thyroglossal duct. No similar case - either malignant or benign - has been reported in the English-language literature. Moreover, in this case the patient had been aware of the mass since the age of 12 years, even though surgery was not performed until she was 21 years old. The patient was young and had no history of radiation exposure. Koike et al. analyzed the incidence of thyroid cancer based on 11,104 cases registered by the Japanese Committee for Registration of Malignant Neoplasms of the Thyroid from 1977 through $1986^{3}$. Most of the patients with follicular carcinomas were in their $50 \mathrm{~s}$ to $60 \mathrm{~s}$, and 
children and adolescents were rare. Follicular carcinomas occurring in the second decade of life accounted for only 44 cases (41 in females and 3 in males).

In the present case, the most difficult aspect was how to treat and adequately follow up this young patient, in view of the few previously reported cases of follicular carcinoma arising from the pyramidal lobe. The guidelines in Europe and the United States recommend that differentiated thyroid carcinoma in adults be treated initially with total thyroidectomy, followed by radioactive iodine ablation ${ }^{4-6}$. Grigsby et al. have reported that thyroid cancer in children and adolescents have a low risk of mortality but a high risk of recurrence and that thyroid capsule invasion, soft tissue invasion, positive margins, and tumor location at diagnosis are significant factors for recurrence ${ }^{7}$. They also stated that total thyroidectomy, postoperative thyroid hormone suppressive therapy, and administration of ${ }^{131} \mathrm{I}$ after intial surgery were performed in a high proportion of cases even when the tumor was confined to the thyroid gland. However, as the histologic subtype of thyroid cancer most frequently affecting children and adolescents is papillary cancer, such previously reported cases may not provide data that are relevant to the treatment and prognosis of follicular cancer. Some studies have found that the rate of recurrence in children with follicular cancer is lower than that in children with papillary cancer ${ }^{8.9}$. Moreover one such study also found that aggressive surgery does not prevent recurrence and is associated with an increased risk of complications. Thus the management of follicular cancer in children and adolescents is controversial. Some studies have reported that patients with minimally invasive follicular cancers have an excellent prognosis even if they underwent thyroid lobectomy ${ }^{10,11}$. Furthermore, most patients with minimally invasive folicullar cancers who were treated with thyroid lobectomy in our hospital (43 cases, average follow-up of 12.5 years) had no recurrence. Therefore, we chose pyramidal lobectomy for this case of mininally invasive follicular cancer in pyramidal lobe. We plan to follow up this patient carefully every 3 months for recurrence in the remnant thyroid gland or metastasis to cervical lymph nodes or distant organs, especially the lung and bone. Follicular carcinoma arising from the pyramidal lobe of the thyroid in adolescents is extremely rare. Therefore, this case suggests the importance of the differential diagnosis of midline cervical masses and the management of this type of neoplasm in adolescents.

\section{References}

1. Braun EM, Windisch G, Wolf G, Hausleithner L, Anderhuber F: The pyramidal lobe: clinical anatomy and its importance in thyroid surgery. Surg Radiol Anat 2007; 29: 21-27.

2. Sturniolo G, Bonanno L, Gagliano E, et al: The thyroid pyramidal lobe: frequency, morphological features and related diseases. Chir Ital 2008; 60: 4146.

3. Koike A, Naruse T: Incidence of thyroid cancer in Japan. Semin Surg Oncol 1991; 7: 107-111.

4. Harris PE: The management of thyroid cancer in adults: a review of new guidelines. Clin Med 2002; 2: 44-46.

5. Singer PA, Cooper DS, Daniels GH, et al.: Treatment guidelines for patients with thyroid nodules and well-differentiated thyroid cancer. American Thyroid Association. Arch Inter Med 1996; 156: 2165-2172.

6. Cooper DS, Doherty GM, Haugen BR, et al.: The American Thyroid Association Guidelines Taskforce: Manegement guidelines for patients with thyroid nodules and differentiated thyroid cancer. Thyroid 2006; 16: 109-142.

7. Grigsby PW, Gal-or A, Michalski JM, Doherty GM: Childhood and adolescent thyroid carcinoma. Cancer 2002; 95: 724-729.

8. La Quaglia MP, Corbally MT, Heller G, Exelby PR, Brennan MF: Recurrence and morbidity in differentiated thyroid carcinoma in children. Surgery 1988; 104: 1149-1156.

9. Schlumberger M, De Vathaire F, Travagli JP, et al: Differentiated thyroid carcinoma in childhood: long term follow-up of 72 patients. J Clin Endocrinol Metab 1987; 65: 1088-1094.

10. Saadi H, Kleidermacher P, Esselstyn C Jr: Conservative management of patients with intrathyroidal well-differentiated follicular thyroid carcinoma. Surgery 2001; 130: 30-35.

11. Wu YS, Wang XD, Zhang WC: Operation strategy for follicular thyroid carcinoma. Ai Zheng 2008; 27: 170-173.

(Received, February 16, 2009)

(Accepted, March 5, 2009) 